\title{
Altered metabolic pathways elucidated via untargeted in vivo toxicometabolomics in rat urine and plasma samples collected after controlled application of a human equivalent amphetamine dose
}

\author{
Selina Hemmer ${ }^{1} \cdot$ Lea Wagmann $^{1} \cdot$ Markus R. Meyer $^{1}$ (D)
}

Received: 2 June 2021 / Accepted: 11 August 2021 / Published online: 19 August 2021

(c) The Author(s) 2021

\begin{abstract}
Amphetamine is widely consumed as drug of abuse due to its stimulating and cognitive enhancing effects. Since amphetamine has been on the market for quite a long time and it is one of the most commonly used stimulants worldwide, to date there is still limited information on its effects on the metabolome. In recent years, untargeted toxicometabolomics have been increasingly used to study toxicity-related pathways of such drugs of abuse to find and identify important endogenous and exogenous biomarkers. In this study, the acute effects of amphetamine intake on plasma and urinary metabolome in rats were investigated. For this purpose, samples of male Wistar rats after a single dose of amphetamine $(5 \mathrm{mg} / \mathrm{kg})$ were compared to a control group using an untargeted metabolomics approach. Analysis was performed using normal and reversed phase liquid chromatography coupled to high-resolution mass spectrometry using positive and negative ionization mode. Statistical evaluation was performed using Welch's two-sample $t$ test, hierarchical clustering, as well as principal component analysis. The results of this study demonstrate a downregulation of amino acids in plasma samples after amphetamine exposure. Furthermore, four new potential biomarkers $\mathrm{N}$-acetylamphetamine, $\mathrm{N}$-acetyl-4-hydroxyamphetamine, $\mathrm{N}$-acetyl4-hydroxyamphetamine glucuronide, and amphetamine succinate were identified in urine. The present study complements previous data and shows that several studies are necessary to elucidate altered metabolic pathways associated with acute amphetamine exposure.
\end{abstract}

Keywords Untargeted metabolomics · Toxicometabolomics $\cdot$ Amphetamine $\cdot$ LC-HRMS/MS

\section{Introduction}

Once introduced as a treatment against narcolepsy, mild depression, post-encephalitic parkinsonism, and several other disorders (Heal et al. 2013), amphetamine nowadays has a limited therapeutic use but is widely consumed as a drug of abuse (DOA) due to its stimulating properties (Carvalho et al. 2012). In 2018, amphetamine was one of the world's most commonly used stimulants, along with cocaine and methamphetamine (UNODC 2020). In addition to the desired effects such as feelings of energy, sociability, and confidence, many adverse effects including hypertension,

Markus R. Meyer

markus.meyer@uks.eu

1 Department of Experimental and Clinical Toxicology, Center for Molecular Signaling (PZMS), Institute of Experimental and Clinical Pharmacology and Toxicology, Saarland University, 66421 Homburg, Germany tachycardia, anxiety, paranoia or auditory and visual hallucinations are associated with its use (Bonisch and Bruss 2006; Steinkellner et al. 2011). These effects are based on its pharmacological ability to act as an indirect sympathomimetic and to increase the release of different neurotransmitters such as noradrenaline and dopamine and/or inhibit their respective reuptake transporter in the presynaptic membrane (Carvalho et al. 2012; de la Torre et al. 2004). Although amphetamine is consumed since decades, there is still little knowledge available regarding its effects on the metabolic state of the organism (Steuer et al. 2020). Conventional in vitro toxicological studies, e.g., using human dopaminergic differentiated SH-SY5Y cells revealed a neurotoxic effect, which caused mitochondrial dysfunction at a concentration of $3.5 \mathrm{mM}$ (Carvalho et al. 2012; Feio-Azevedo et al. 2017). 
Metabolomics in general is used for discovery of novel biomarkers, investigation of physiologic status, or identification of perturbed biochemical pathways (Nicholson and Lindon 2008) and can provide a snapshot analysis of the whole metabolome in a biological system (Liu and Locasale 2017). Toxicometabolomics, a sub-discipline of metabolomics, is dedicated to elucidate the pattern of small molecules (usually below $1500 \mathrm{Da}$ ) within an in vitro or in vivo system related to a certain stimulus such as DOA intake. Under highly controlled study conditions, changes of the metabolome can be observed that may indicate or be the result of a certain drug intake (Wang et al. 2016). Toxicometabolomics can, therefore, be used to study toxicity-related pathways such as the mode of action of xenobiotics or in screening of drug induced cellular or organ toxicity, or to discover new biomarkers (Bouhifd et al. 2013; Ramirez et al. 2013). In recent years, toxicometabolomics have been increasingly used in the field of DOA (Araujo et al. 2021; Manier et al. 2020a, b; Steuer et al. 2020; Zaitsu et al. 2016). Its application may allow to find exogenous biomarkers, which could be new drug metabolites, and on the other hand to identify endogenous biomarkers, which could not only be indications of acute drug ingestion or sample manipulation but also provide information in the mechanism of drug action, consumption behavior, or can be used to assess the severity of intoxications (Steuer et al. 2019; Wang et al. 2016). Steuer et al. (2020) investigated changes of the plasma metabolome after amphetamine intake in a controlled human study of 13 participants and identified an increased energy and steroid metabolism. However, since there is no method that can reveal the complete metabolome and since the plasma metabolome is highly dynamic and influenced by various factors, further studies are needed. In vivo studies in laboratory animals are suitable for this purpose. Under wellstandardized and comparable conditions such as controlled diet, sleep cycles and little genetic variability, it is possible to better delineate the metabolome changes caused by amphetamine use. Furthermore, to the best of our knowledge, there are no studies on the urinary metabolome after amphetamine exposure available.

This study should provide the metabolic profiling of rat plasma and urine in response to acute amphetamine exposure, provide additional metabolites/biomarker in urine for detection of amphetamine intake and should complement previous studies. Data should allow to observe changes in the metabolome caused by amphetamine and allow to identify biological pathways affected by its intake, which are necessary to further understand its acute and chronic effects and support further targeted analysis. The analysis should be done by liquid chromatography coupled to high-resolution tandem mass spectrometry (LC-HRMS/MS).

\section{Materials and methods}

\section{Chemicals and reagents}

Racemic D-/L-amphetamine sulfate was purchased from Lipomed (Weil am Rhein, Germany). Acetonitrile, ethanol, and methanol (all LC-MS grade) were obtained from VWR (Darmstadt, Germany), ammonium formate, ammonium acetate, and formic acid, amino acids standards solution, D-Glucose-1,2,3,4,5,6,6- $\mathrm{d}_{7}$, palmitic acid- $\mathrm{d}_{31}$, and creatinine$\mathrm{d}_{3}$ from Merck (Darmstadt, Germany). L-Tryptophan- $\mathrm{d}_{5}$ was obtained from Alsachim (Illkirch-Graffenstaden, France). Water was purified with a Millipore filtration unit (18.2 $\Omega \times$ cm water resistance).

\section{Study design}

Ten adolescent male Wistar rats (Charles River, Sulzfeld, Germany) were housed in a controlled environment (temperature $22{ }^{\circ} \mathrm{C}$, humidity $57 \pm 2 \%$, and $12 \mathrm{~h} \mathrm{light/dark}$ cycles). Studies have been approved by an ethics committee (33/2019-Landesamt für Verbraucherschutz, Saarbrücken, Germany). A single dose of $5 \mathrm{mg} / \mathrm{kg}$ body weight (BW) racemic $\mathrm{D}-/ \mathrm{L}$-amphetamine was administered as aqueous suspension by gastric intubation to five rats. Five control rats were administrated only with water. During the study, rats were housed in metabolism cages for $24 \mathrm{~h}$, having water ad libitum. Animal general health aspects were assessed at the time points $30 \mathrm{~min}, 60 \mathrm{~min}, 120 \mathrm{~min}, 360 \mathrm{~min}$, and $24 \mathrm{~h}$ after amphetamine intake. The animals were then monitored including only some general aspects such as body weight, clean orifices, clear eyes, and sleep behavior. Detailed changes expected after intake of stimulants such as heart rate, radial maze for cognitive function or plus maze to determine activity and anxiety behavior were not and could not be monitored as this was not the focus of the current study.

The selected dose of $5 \mathrm{mg} / \mathrm{kg} \mathrm{BW}$ D-/L-amphetamine is equivalent to $50 \mathrm{mg}$ in a $60 \mathrm{~kg}$ human according to the allometric scaling principles of Nair and Jacob (2016). This would correspond to a human D-amphetamine dose of $25 \mathrm{mg}$, which is in line with the work by Dolder et al. (2017) and $50 \mathrm{mg}$ of a racemic mixture, which is used as recreational drug (http://psychoaktivesubstanzen.de/amphetamin. Accessed 26-May-2020, 9:30).

\section{Sample collection}

Urine was collected separately from the feces over a period of eight or $24 \mathrm{~h}$ after administration, aliquoted, and frozen at $-80^{\circ} \mathrm{C}$ until use. Blood samples were collected 1,2, and 
$8 \mathrm{~h}$ after administration. For blood sampling, animals were anesthetized with diethyl ether and blood was withdrawn from the Vena caudalis mediana using a heparin-coated syringe. Blood samples were centrifuged (1503 rcf, $5 \mathrm{~min}$, $24{ }^{\circ} \mathrm{C}$ ), and plasma was removed and immediately stored at $-80{ }^{\circ} \mathrm{C}$ until analysis.

\section{Sample preparation}

According to Manier and Meyer (2020), plasma samples were prepared as follow. A volume of $50 \mu \mathrm{L}$ plasma was transferred into a reaction tube and precipitated using $200 \mu \mathrm{L}$ of a mixture of methanol and ethanol $(1: 1, \mathrm{v} / \mathrm{v})$. The mixture contained $48 \mu \mathrm{M}$ L-tryptophan- $\mathrm{d}_{5}, 8.6 \mu \mathrm{M}$ creatinine- $\mathrm{d}_{3}, 34.8 \mu \mathrm{M}$ palmitic acid- $\mathrm{d}_{31}$, and $53.4 \mu \mathrm{M}$ D-glucose$\mathrm{d}_{7}$ as internal standards. Samples were shaken for $2 \mathrm{~min}$ at $2000 \mathrm{rpm}$ and subsequently centrifuged for $30 \mathrm{~min}$ at 21,130 rcf and $2{ }^{\circ} \mathrm{C} .150 \mu \mathrm{L}$ of the supernatant was transferred into a new reaction tube and evaporated to dryness using a vacuum centrifuge at $1400 \mathrm{rpm}$ and $24{ }^{\circ} \mathrm{C}$ for $20 \mathrm{~min}$. The obtained residues were reconstituted in $50 \mu \mathrm{L}$ of a mixture of acetonitrile and methanol $(70: 30, \mathrm{v} / \mathrm{v})$.

In accordance with Barnes et al. (2016), urine samples were centrifugated at $13,523 \mathrm{rcf}$ at $4{ }^{\circ} \mathrm{C}$ for $10 \mathrm{~min}$ to remove any precipitates. $50 \mu \mathrm{L}$ of urine were transferred in a reaction tube and $200 \mu \mathrm{L}$ methanol including $48 \mu \mathrm{M}$ L-tryptophan- $\mathrm{d}_{5}, 8.6 \mu \mathrm{M}$ creatinine- $\mathrm{d}_{3}, 34.8 \mu \mathrm{M}$ palmitic acid- $\mathrm{d}_{31}$, and $53.4 \mu \mathrm{M}$ D-glucose- $\mathrm{d}_{7}$ as internal standards were added. Samples were cooled to $-20^{\circ} \mathrm{C}$ for $20 \mathrm{~min}$ and then centrifugated for $10 \mathrm{~min}$ at $13,523 \mathrm{rcf}$ and $4{ }^{\circ} \mathrm{C} .150 \mu \mathrm{L}$ of the supernatant were transferred into a new reaction tube and evaporated to dryness using a vacuum centrifuge at $1400 \mathrm{rpm}$ and $24{ }^{\circ} \mathrm{C}$. The obtained residues were reconstituted in $50 \mu \mathrm{L}$ of a mixture of acetonitrile and methanol $(70: 30, v / v)$.

For each matrix and the corresponding timepoint, one pooled quality control (QC) sample was prepared by transferring $10 \mu \mathrm{L}$ of each sample into one MS vial. These QC samples were also used for optimization of the peak picking parameters and identification of significant features, as described below (QC group).

\section{LC-HRMS/MS apparatus}

According to Manier et al. (2019b), analyses were performed using a Thermo Fisher Scientific (TF, Dreieich, Germany) Dionex UltiMate 3000 RS pump consisting of a degasser, a quaternary pump, and an UltiMate Autosampler, coupled to a TF Q-Exactive Plus system including a heated electrospray ionization (HESI)-II source. Performance of the columns and the mass spectrometer was tested using a test mixture as described by Maurer et al. (Maurer et al. 2018, 2016). Gradient reversed phase (RP) elution was performed on a TF Accucore Phenyl-Hexyl column $(100 \mathrm{~mm} \times 2.1 \mathrm{~mm}, 2.6 \mu \mathrm{m})$ and normal phase (NP) elution using a Macherey-Nagel (Düren, Germany) HILIC Nucleodur column $(125 \mathrm{~mm} \times 3 \mathrm{~mm}, 3 \mu \mathrm{m})$. The mobile phase and gradient for the Phenyl-Hexyl column consisted of $2 \mathrm{mM}$ aqueous ammonium formate containing acetonitrile $(1 \%, \mathrm{v} / \mathrm{v})$ and formic acid $(0.1 \%, \mathrm{v} / \mathrm{v}, \mathrm{pH}$ 3 , eluent $\mathrm{A}$ ), as well as $2 \mathrm{mM}$ ammonium formate solution with acetonitrile:methanol $(1: 1, \mathrm{v} / \mathrm{v})$ containing water $(1 \%, \mathrm{v} / \mathrm{v})$ and formic acid $(0.1 \%, \mathrm{v} / \mathrm{v}$, eluent B). The flow rate was set from 1 to $10 \mathrm{~min}$ to $500 \mu \mathrm{L} / \mathrm{min}$ and from 10 to $13.5 \mathrm{~min}$ to $800 \mu \mathrm{L} / \mathrm{min}$ using the following gradient: 0-1 min hold $99 \%$ A, 1-10 min to $1 \%$ A, 10-11.5 min hold $1 \%$ A, 11.5-13.5 min hold 99\% A. The gradient elution for normal phase chromatography was performed using aqueous ammonium acetate $(200 \mathrm{mM}$, eluent $\mathrm{C})$ and acetonitrile containing formic acid $(0.1 \%$, v/v, eluent D). The flow rate was set to $500 \mu \mathrm{L} / \mathrm{min}$ using the following gradient: 0-1 min hold $2 \% \mathrm{C}, 1-5 \mathrm{~min}$ to $20 \% \mathrm{C}, 5-8.5 \mathrm{~min}$ to $60 \% \mathrm{C}, 8.5-10$ min hold $60 \% \mathrm{C}, 10-12$ min hold $2 \%$ C. For preparation and cleaning of the injection system, isopropanol:water $(90: 10, \mathrm{v} / \mathrm{v})$ was used. The following settings were used: wash volume, $100 \mu \mathrm{L}$; wash speed, $4000 \mathrm{~nL} / \mathrm{s}$; loop wash factor, 2 . Column temperature for every analysis was set to $40{ }^{\circ} \mathrm{C}$, maintained by a Dionex UltiMate $3000 \mathrm{RS}$ analytical column heater. Injection volume was set to $1 \mu \mathrm{L}$. HESI-II source conditions were as follows: ionization mode, positive or negative; sheath gas, $60 \mathrm{AU}$; auxiliary gas, $10 \mathrm{AU}$; sweep gas, $3 \mathrm{AU}$; spray voltage, $3.5 \mathrm{kV}$ in positive and $-4.0 \mathrm{kV}$ in negative mode; heater temperature, $320{ }^{\circ} \mathrm{C}$; ion transfer capillary temperature, $320^{\circ} \mathrm{C}$; and S-lens RF level, 50.0. Mass spectrometry for untargeted metabolomics was performed according to a previously optimized workflow (Manier et al. 2019a, b). The settings for full scan (FS) data acquisition were as follows: resolution, 140,000 fwhm; microscan, 1; automatic gain control (AGC) target, $5 \times 10^{5}$; maximum injection time, $200 \mathrm{~ms}$; scan range, $\mathrm{m} / \mathrm{z}$ 50-750; spectrum data type; centroid. All study samples were analyzed in randomized order, to avoid potential analyte instability or instrument performance to confound data interpretation. Additionally, one QC injection was performed every five samples to monitor batch effects, as described by Wehrens et al. (Wehrens et al. 2016).

Significant features were subsequently identified using PRM. Settings for PRM data acquisition were as follow: resolution, 35,000 fwhm; microscans, 1; AGC target, $5 \times 10^{5}$; maximum injection time, $200 \mathrm{~ms}$; isolation window, $1.0 \mathrm{~m} / z$; collisions energy (CE), 10, 20,35, or $40 \mathrm{eV}$; spectrum data type, centroid. The inclusion list contained the monoisotopic masses of all significant features and a time window of their retention time $\pm 60 \mathrm{~s}$. TF Xcalibur software version 3.0.63 was used for data handling. 


\section{Data processing and statistical analysis}

Thermo Fisher LC-HRMS/MS RAW files were converted into mzXML files using ProteoWizard (Adusumilli and Mallick 2017). Optimization of XCMS parameter was done on a previously optimized strategy as mentioned by Manier et al. (2019a). Peak picking and alignment parameters are summarized in Table $\mathrm{S} 1$ in the supplementary data. Peak picking was performed using XCMS in an R environment (Smith et al. 2006; Team) and the R package CAMERA (Kuhl et al. 2012) was used for the annotation of adducts, artifacts, and isotopes. Feature abundance with a value of zero were replaced by the lowest measured abundance as a surrogate limit of detection and the whole dataset was subsequently $\log 10$ transformed (Wehrens et al. 2016). Normalization was performed for urine samples using the area of endogenous creatinine from those samples analyzed using normal phase column and positive ionization mode and for plasma samples using the internal standard L-tryptophan- $\mathrm{d}_{5}$. Significant changes of features between control and amphetamine group were assumed after evaluating their fold change using a threshold of 1.5, as well as after Welch's two-sample $t$ test and a $p$ value $<0.025$. Principal component analysis (PCA) and hierarchical clustering were used to investigate patterns in the datasets. Names for the features were adopted from XCMS using "M" followed by rounded mass and "T" followed by the retention time in seconds. After visual inspection of the extracted ion chromatograms (EIC) of significant features, the significant features were divided into true and false features based on the peak shape quality of their EIC (Hemmer et al. 2020). The R scripts and the mzXML files can be found at https://github.com/sehem/Amphetamine_ Metabolomics.git.

\section{Identification of significant features}

Significant features were identified by recording MS/MS spectra using the PRM method mentioned above. After conversion to mzXML format using ProteoWizard (Adusumilli and Mallick 2017), spectra were imported to NIST MSSEARCH version 2.3. Library search for identification was performed using the following settings: spectrum search type, identity (MS/MS); precursor ion $\mathrm{m} / \mathrm{z}$, in spectrum; spectrum search options, none; presearch, off; other options, none. MS/MS search was conducted using the following settings: precursor tolerance, $\pm 5 \mathrm{ppm}$; product ion tolerance, $\pm 10 \mathrm{ppm}$; ignoring peaks around precursor, $\pm \mathrm{m} / \mathrm{z}$ 1 (Manier et al. 2020b). Following libraries were used: NIST 2014 (nist_msms and nist_msms2 sublibrary) (Linstrom and Mallard 2001), Wiley METLIN Mass Spectral Database (Guijas et al. 2018), LipidBlast (Kind et al. 2013), MMHW (Maurer et al. 2018), the Human Metabolome Database (Wishart et al. 2007) (HMDB, V4.0). Metabolites of amphetamine were tentatively identified by interpreting their spectra in comparison to that of the parent compound. The in-silico fragmentation tool MetFrag (https://msbi.ipbhalle.de/MetFrag/) was applied to MS/MS data to identify potential substructures. Identified features were classified on the different levels of identification according to the Metabolomics Standards Initiative (MSI) (Sumner et al. 2007): affirmation using MS/MS information and co-elution with authentic standards (level 1), affirmation without chemical reference standards, based on comparison of experimental MS/MS spectra with public/commercial spectral libraries (level 2), annotation of putatively characterized compound classes based on characteristic physicochemical properties of a chemical class of compounds, or by spectral similarity to known compounds of a chemical class (level 3), and unidentified or unclassified metabolites (level 4).

\section{Metabolic pathway analysis}

To identify the endogenous metabolic pathways affected by amphetamine intake, all compounds identified with level 1 were imported to MetaboAnalyst 5.0 (http://www.metab oanalyst.ca) and searched against Rattus norvegicus metabolite database, for each matrix and time points. Scatter plot was selected as visualization method and the hypergeometric test with the relative-betweenness centrality algorithm was used. For further biological interpretation biochemical pathways with a significant level of $p<0.05$ was used.

\section{Results}

Data files in mzXML format and the corresponding $\mathrm{R}$ files can be found at https://github.com/sehem/Amphetamine_ Metabolomics.git. Results of univariate and multivariate statistic as well as the $\mathrm{MS}^{2}$ spectra of amphetamine metabolites are available as supplementary data.

\section{Animal general health aspects}

Amphetamine exposed animals in this study showed no effect on their stereotyped behavior or exploratory activity after administration. Furthermore, no significant body weight loss could be observed in comparison to the control group.

\section{Untargeted metabolomics: univariate and multivariate statistics}

Volcano plots of detected features are shown in Fig. S1-4. An overview of the total number of significant features and their percentage of adducts/artifacts, isotopes, and falsepositive results are shown in Table S2. In addition, datasets 
were analyzed using multivariate methods in form of PCA and hierarchical clustering, to identify the largest changing features and specific signatures. Results of the hierarchical clustering which are displayed in heatmaps are shown in Fig. S5-8. Results of the scores of PCA of all matrices and time points are shown in Fig. S9-12.

\section{Plasma}

Using the four different analytical methods (RP positive, RP negative, NP positive, NP negative), 41 features were found in total to be significant at all three plasma time points after amphetamine administration. Plasma samples which were taken $1 \mathrm{~h}$ after administration, revealed 14 significant features after using RP and NP and positive ionization mode, which contained one isotope and two adducts according to CAMERA. However, one of these significant features was manually marked as false-positive, due to its EIC showing a poor peak shape quality. Analyses using RP and NP and negative ionization mode did not reveal any significant changes at that time point. Considering the heat maps, a clear separation between the control group and the amphetamine group is shown by NP (Fig. S5a). The dataset of the plasma samples which were taken $2 \mathrm{~h}$ after administration, revealed 13 significant features. These features included nine false-positive features, as well as two isotopes. Again, using $\mathrm{RP}$ and negative ionization did not reveal any significant features. Looking at the PCA, the two groups amphetamine and control measured in positive ionization mode separated well (Fig. S9b and S11b). In plasma samples received $8 \mathrm{~h}$ after administration, 18 significant features were observed only in positive ionization mode. These features included five false-positive hits and two isotopes. Both heatmaps showed a clear separation of the amphetamine and control group (Fig. S5c and S7c).

\section{Urine}

In urine samples, 88 significant features were found in total using the above mentioned four different analytical methods in the samples collected after 8 and $24 \mathrm{~h}$. Sixty-four significant features were found in the 8-h urine samples. These features included 18 false-positive hits, as well as five isotopes and seven artifacts according to CAMERA. Heatmaps showed a good clustering of all groups (Fig. S5d, S6b, S7d, and S8a). Furthermore, in comparison to plasma, amphetamine samples are clustered very closely together in the PCA scores, whereas the control group appears more distributed (Fig. S9d, S10b, S11d, and S12a). Urine samples which were collected $24 \mathrm{~h}$ after administration revealed 32 significant features. These features included two false-positive hits, two isotopes, and three artifacts. The four heatmaps displayed a good clustering of the groups (Fig. S5e, S6c, S7e, and S8b).
In comparison to the PCA scores after $8 \mathrm{~h}$, the amphetamine group appears more distributed after $24 \mathrm{~h}$.

\section{Identification of significant features}

The results of the identification of significant features are summarized in Tables 1 and 2. The given level of identification was in accordance with the MSI (Sumner et al. 2007). Isotopes that were putatively identified by CAMERA were not further identified. No $\mathrm{MS}^{2}$ spectra could be recorded for several features due to their low abundance.

\section{Plasma}

In total, 14 compounds could be identified with a level of 1 or 2 (Table 1 ). $1 \mathrm{~h}$ after administration, most identified compounds were amino acids, which could all be identified with level 1 according to MSI. Additionally, the sesquiterpenoid tocopheronic acid was identified. In comparison to the control group all compounds were downregulated. Amphetamine and its metabolite $\mathrm{N}$-acetylamphetamine were identified in samples drawn $2 \mathrm{~h}$ after administration. Furthermore, erucamide, an unsaturated fatty amide was upregulated compared to the control group. In plasma samples obtained after the $8 \mathrm{~h}$, the identified compounds were again amino acids and $\mathrm{N}$-acylsphingosines such as L-methionine and ceramide. While amounts of most amino acids were decreased compared to control group, all other compounds had increased.

\section{Urine}

Table 2 summarizes the 21 compounds which were identified in urine samples. Compared to urine collected after8 h, only amphetamine and its metabolites could be identified in the 24-h urine samples, except for $\mathrm{N}$-acetylhistamine. Most of the identified compounds in 8-h urine samples were either amino acids or amphetamine metabolites. All identified compounds had increased in comparison to the control group except for L-tryptophan and spermidine.

\section{Metabolic pathway analysis}

since no substances with a level of 1 were identified in plasma samples $2 \mathrm{~h}$ after amphetamine administration, only the scatter plots of 1- and 8-h plasma samples are shown in Fig. 1a, b. The identified metabolic pathway in plasma samples $1 \mathrm{~h}$ after administration with $p<0.05$ were aminoacyltRNA biosynthesis, phenylalanine, tyrosine, and tryptophan biosynthesis, valine, leucine, and isoleucine biosynthesis, and ubiquinone and other terpenoid-quinone biosynthesis. For the 8-h plasma samples, glycine, serine, and threonine metabolism, aminoacyl-tRNA biosynthesis, and valine, leucine, and isoleucine biosynthesis were found as significantly 
Table 1 Identified compounds in plasma samples that showed significant changes between amphetamine (A) and control (C) group, sorted according to compound classes, $m / z$ values are given for the highest prevalent ion species

\begin{tabular}{|c|c|c|c|c|c|c|c|c|c|}
\hline Compound name & $\begin{array}{l}\text { Identification } \\
\text { level }\end{array}$ & Compound class & $m / z$ & Chromatography & Adducts & Change & $\begin{array}{l}p(1 \mathrm{~h}, \\
\text { A vs. } \\
\text { C) }\end{array}$ & $\begin{array}{l}p(2 \mathrm{~h}, \\
\text { A vs. } \\
\text { C) }\end{array}$ & $\begin{array}{l}p(8 \mathrm{~h}, \\
\text { A vs. } \\
\text { C) }\end{array}$ \\
\hline Creatine & 1 & Amino acid & 131.0695 & $\mathrm{RP}$ & $\mathrm{M}+\mathrm{H}$ & $\uparrow$ & n.s & n.s & $*$ \\
\hline L-Tryptophan & 1 & Amino acid & 204.0899 & $\mathrm{RP}$ & $\begin{array}{l}\mathrm{M}+\mathrm{H} \\
\mathrm{M}+\mathrm{H}-\mathrm{NH} 3 \\
\mathrm{M}+\mathrm{K}^{*} \mathrm{HCOOH} \\
\mathrm{M}+1\end{array}$ & $\downarrow$ & $* *$ & n.s & n.s \\
\hline L-Citrulline & 1 & Amino acid & 175.0957 & NP & $\mathrm{M}+\mathrm{H}$ & $\downarrow$ & $*$ & n.s & n.s \\
\hline L-Histidine & 1 & Amino acid & 155.0695 & $\mathrm{RP}$ & $\mathrm{M}+\mathrm{H}$ & $\downarrow$ & $* *$ & n.s & n.s \\
\hline L-Methionine & 1 & Amino acid & 149.0510 & $\mathrm{RP}, \mathrm{NP}$ & $\mathrm{M}+\mathrm{H}$ & $\downarrow$ & $* *$ & n.s & $* *$ \\
\hline L-Proline & 1 & Amino acid & 115.0633 & $\mathrm{RP}, \mathrm{NP}$ & $\mathrm{M}+\mathrm{H}$ & $\downarrow$ & $*$ & n.s & n.s \\
\hline L-Threonine & 1 & Amino acid & 119.0582 & $\mathrm{RP}, \mathrm{NP}$ & $\mathrm{M}+\mathrm{H}$ & $\downarrow$ & $* *$ & n.s & $*$ \\
\hline L-Tyrosine & 1 & Amino acid & 181.0739 & $\mathrm{RP}, \mathrm{NP}$ & $\mathrm{M}+\mathrm{H}$ & $\downarrow$ & $*$ & n.s & n.s \\
\hline Amphetamine & 1 & Amphetamine & 135.1048 & $\mathrm{RP}$ & $\mathrm{M}+\mathrm{H}$ & $\uparrow$ & n.s & $*$ & n.s \\
\hline $\begin{array}{l}\text { Amphetamine-M } \\
(N \text {-acetyl })\end{array}$ & 2 (NIST msms) & Amphetamine & 177.1154 & $\mathrm{RP}$ & $\mathrm{M}+\mathrm{H}$ & $\uparrow$ & n.s & $* *$ & n.s \\
\hline $\begin{array}{l}\text { Ceramide } \\
(\mathrm{d} 18: 1 / 23: 0)\end{array}$ & 2 (Lipidmaps) & $\begin{array}{l}N \text {-acylsphingo- } \\
\text { sine }\end{array}$ & 635.6216 & NP & $\mathrm{M}+\mathrm{H}, \mathrm{M}+1$ & $\uparrow$ & n.s & n.s & $* *$ \\
\hline Nicotinamide & 2 (NIST ms/ms) & $\begin{array}{l}\text { Pyridine carbox- } \\
\text { ylic acids }\end{array}$ & 122.0480 & NP & $\mathrm{M}+\mathrm{H}$ & $\downarrow$ & n.s & n.s & $*$ \\
\hline $\begin{array}{l}\text { Tocopheronic } \\
\text { acid }\end{array}$ & 3 (hmdb) & Sesquiterpenoids & 294.1467 & NP & $\mathrm{M}+\mathrm{H}-\mathrm{H} 2 \mathrm{O}$ & $\downarrow$ & $* *$ & n.s & n.s \\
\hline Erucamide & 2 (NIST msms) & $\begin{array}{l}\text { Unsaturated fatty } \\
\text { amide }\end{array}$ & 337.3345 & NP & $\mathrm{M}+\mathrm{H}, \mathrm{M}+1$ & $\uparrow$ & n.s & $*$ & n.s \\
\hline
\end{tabular}

Identification levels for each metabolite are given according to MSI (Sumner et al. 2007). The corresponding chromatography method is given for normal phase (NP) and for reversed phase (RP) chromatography. Statistical was performed by Welch $t$ test $(p<0.025)$ : not significant (n.s.) $>0.025$

$* 0.01-0.025$

$* * 0.001-0.01$

$* * *<0.001$

changed metabolic pathways. In the 8-h urine samples, only two endogenous metabolites were identified by level 1 , therefore, the scatter plot shows only one significant hit for arginine biosynthesis (Fig. 1c). No endogenous metabolites could be identified with level 1 according to MSI in urine $24 \mathrm{~h}$ after administration and, therefore, no metabolic pathway analysis was possible.

\section{Discussion}

The metabolome is considered as all compounds with molecular weights less than $1500 \mathrm{Da}$, which could be detected in, e.g., biofluids or tissues (Barnes et al. 2016). These molecules are not necessarily originating from the biological sample but also from, e.g., tubing vials and reagents. Samples such as plasma or urine are particularly complex since the metabolome is affected additionally by, e.g., food, microbiome, and drugs used to anesthetize experimental animals
(Barnes et al. 2016). Since there are many parameters, which can influence the human metabolome, animal models are well suited to study changes in the metabolome, as they are less complex than human studies and can be performed under standardized and comparable conditions. Animals are subject to a uniform sleep-wake rhythm, kept under the same conditions, receive the same food and water, and they have the advantage that their genetic variability is very low. Furthermore, a metabolomic study requires significantly fewer animals than would be needed in a human clinical study to obtain reliable results. They are also beneficial compared to in vitro studies, which often represent only certain cells or organs and thus only a part of an entire organism. Thus, ten male adolescents Wistar rats were used in this study. Certain metabolites are released or excreted into blood and urine due to a certain stimulus such as drug of abuse intake. There they can be identified and serve as potential biomarkers (Wang et al. 2016). While plasma is primarily of interest in terms of changes in endogenous metabolites 
Table 2 Identified compounds in urine samples that showed significant changes between amphetamine (A) and control (C) group, sorted according to compound classes, $\mathrm{m} / \mathrm{z}$ values are given for the highest prevalent ion species

\begin{tabular}{|c|c|c|c|c|c|c|c|c|}
\hline Compound name & $\begin{array}{l}\text { Identification } \\
\text { level }\end{array}$ & Compound class & $m / z$ & $\begin{array}{l}\text { Chromatogra- } \\
\text { phy }\end{array}$ & Adducts & Change & $\mathrm{p}(8 \mathrm{~h}, \mathrm{~A}$ vs. C) & $\begin{array}{l}\mathrm{p}(24 \mathrm{~h}, \\
\text { A vs. } \\
\text { C) }\end{array}$ \\
\hline $\begin{array}{l}\text { 4-Hydroxy-6-methyl- } \\
\text { 2-pyron }\end{array}$ & 2 (NIST msms) & & 126.0317 & NP & $\mathrm{M}+\mathrm{H}$ & $\uparrow$ & $*$ & n.s \\
\hline Imidazole lactate & 2 (NIST msms) & & 156.0535 & NP & $\mathrm{M}+\mathrm{H}$ & $\uparrow$ & $*$ & n.s \\
\hline Histamine & 2 (NIST msms) & Amines & 111.0796 & NP & $\mathrm{M}+\mathrm{H}$ & $\uparrow$ & $* *$ & n.s \\
\hline $\begin{array}{l}\text { L-Pentahomomethio- } \\
\text { nine }\end{array}$ & 2 (METLIN) & Amino acids & 219.1293 & NP & $\mathrm{M}+\mathrm{H}$ & $\uparrow$ & $*$ & n.s \\
\hline L-Tryptophan & 1 & Amino acids & 204.0899 & $\mathrm{RP}$ & $\mathrm{M}+\mathrm{H}$ & $\downarrow$ & $*$ & n.s \\
\hline $\mathrm{N}$-acetyl-L-arginine & 2 (NIST msms) & Amino acids & 216.1222 & NP & $\mathrm{M}+\mathrm{H}$ & $\uparrow$ & $* *$ & n.s \\
\hline$N$-acetylhistamine & 2 (NIST msms) & Amino acids & 153.0902 & $\mathrm{RP}$ & $\mathrm{M}+\mathrm{H}$ & $\uparrow$ & n.s & $*$ \\
\hline $\begin{array}{l}N^{2}, N^{5} \text {-diacetylorni- } \\
\text { thine }\end{array}$ & 2 (NIST msms) & Amino acids & 216.1110 & $\mathrm{RP}$ & $\mathrm{M}+\mathrm{H}$ & $\uparrow$ & $*$ & n.s \\
\hline Spermidine & 2 (NIST msms) & Amino acids & 145.1579 & $\mathrm{RP}$ & $\mathrm{M}+\mathrm{H}$ & $\downarrow$ & $*$ & n.s \\
\hline $\begin{array}{l}\gamma \text {-Glutamyl- } \gamma \text { - } \\
\text { aminobutyraldehyde }\end{array}$ & 2 (NIST msms) & Amino acids & 216.1110 & NP & M-H & $\uparrow$ & $* *$ & n.s \\
\hline Amphetamine & 1 & Amphetamine & 135.1048 & $\mathrm{RP}, \mathrm{NP}$ & $\begin{array}{l}\mathrm{M}+\mathrm{H}-\mathrm{NH} 3 \\
\mathrm{M}+\mathrm{D}-\mathrm{NH} 3 \\
\mathrm{M}+\mathrm{H}, \mathrm{M}+\mathrm{H}, \\
\mathrm{M}+\mathrm{D} \\
\mathrm{M}+1, \mathrm{M}+2 \\
\mathrm{M}+\mathrm{H}-107\end{array}$ & $\uparrow$ & $* *$ & $* * *$ \\
\hline $\begin{array}{c}\text { Amphetamine-M } \\
\text { (3-OH sulfate) }\end{array}$ & 2 (MMHW) & Amphetamine & 231.0565 & $\mathrm{RP}, \mathrm{NP}$ & $\mathrm{M}+\mathrm{H}$ & $\uparrow$ & $* *$ & $* *$ \\
\hline $\begin{array}{l}\text { Amphetamine-M } \\
\text { (4-hydroxy glucu- } \\
\text { ronide) }\end{array}$ & 3 & Amphetamine & 327.1318 & $\mathrm{RP}$ & $\mathrm{M}+\mathrm{H}$ & $\uparrow$ & $* *$ & n.s \\
\hline $\begin{array}{l}\text { Amphetamine-M } \\
\text { (4-hydroxy-) }\end{array}$ & 3 & Amphetamine & 151.0997 & RP, NP & $\begin{array}{l}\mathrm{M}+\mathrm{H} \\
\mathrm{M}+\mathrm{H}-(107) \\
\mathrm{M}+\mathrm{D}\end{array}$ & $\uparrow$ & $* * *$ & $* *$ \\
\hline $\begin{array}{l}\text { Amphetamine-M } \\
\text { (6-oxohexanoic } \\
\text { acid-) }\end{array}$ & 3 & Amphetamine & 263.1521 & NP & $\mathrm{M}+\mathrm{H}$ & $\uparrow$ & $* * *$ & n.s \\
\hline $\begin{array}{l}\text { Amphetamine-M } \\
\text { ( } N \text {-acetyl-4-hydroxy } \\
\text { glucuronide) }\end{array}$ & 3 & Amphetamine & 369.1424 & $\mathrm{RP}, \mathrm{NP}$ & $\mathrm{M}+\mathrm{H}, \mathrm{H}-\mathrm{H}$ & $\uparrow$ & $* *$ & $* *$ \\
\hline $\begin{array}{l}\text { Amphetamine-M } \\
(N \text {-acetyl- })\end{array}$ & 3 & Amphetamine & 177.1154 & NP & $\mathrm{M}+\mathrm{H}$ & $\uparrow$ & n.s & $*$ \\
\hline $\begin{array}{l}\text { Amphetamine-M } \\
(N \text {-acetyl-4-hy- } \\
\text { droxy- })\end{array}$ & 3 & Amphetamine & 193.1103 & NP & $\mathrm{M}+\mathrm{H}$ & $\uparrow$ & $*$ & n.s \\
\hline $\begin{array}{l}\text { Amphetamine suc- } \\
\text { cinate }\end{array}$ & 3 & Amphetamine & 235.1208 & NP & $\mathrm{M}+\mathrm{H}, \mathrm{M}+\mathrm{D}$ & $\uparrow$ & $* * *$ & $* *$ \\
\hline $\begin{array}{l}\text { 5-Acetylamino- } \\
\text { 6-amino-3-methyl- } \\
\text { uracil }\end{array}$ & 2 (MetFrag) & $N$-arylamides & 198.0753 & NP & $\mathrm{M}+\mathrm{H}, \mathrm{M}+\mathrm{D}$ & $\uparrow$ & $*$ & n.s \\
\hline 1,3-Dimethyluracil & 2 (MetFrag) & Pyrimidines & 140.0586 & NP & $\mathrm{M}+\mathrm{H}, \mathrm{M}+\mathrm{D}$ & $\uparrow$ & $*$ & n.s \\
\hline Urea & 1 & Ureas & 60.0324 & $\mathrm{RP}$ & $\mathrm{M}+\mathrm{Na}$ & $\uparrow$ & $*$ & n.s \\
\hline
\end{tabular}

Identification levels for each metabolite are given according to MSI (Sumner et al. 2007). The corresponding chromatography method is given for normal phase (NP) and for reversed phase (RP) chromatography. Statistical was performed by Welch $t$ test $(p<0.025)$ : not significant (n.s.) $>0.025$

$* 0.01-0.025$

$* * 0.001-0.01$

$* * *<0.001$ 
a

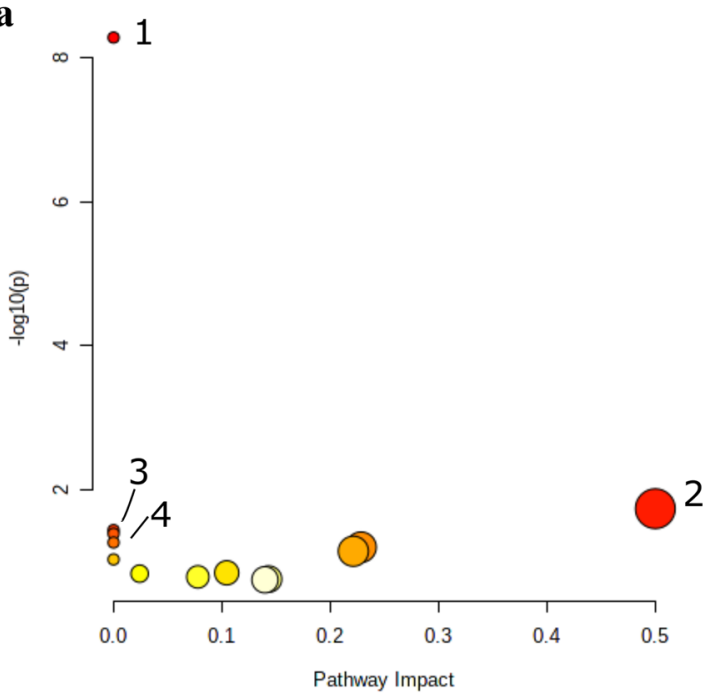

c

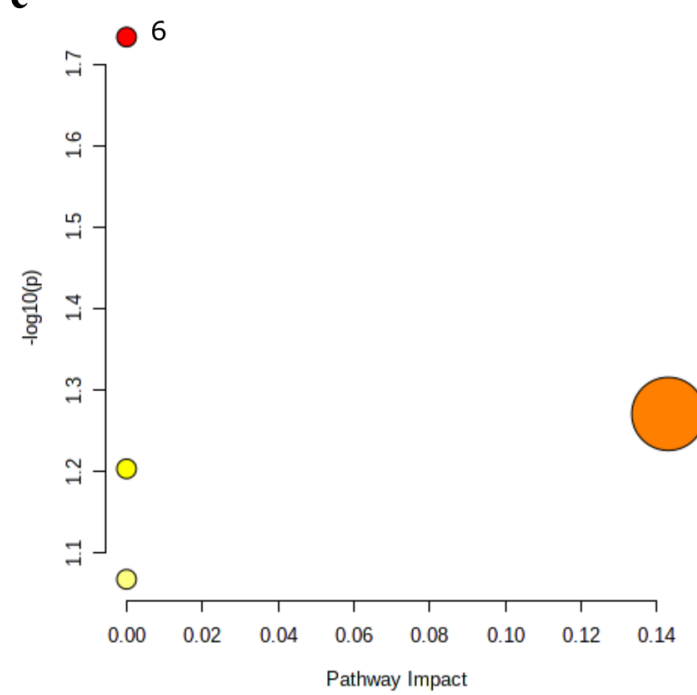

b

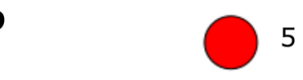

을

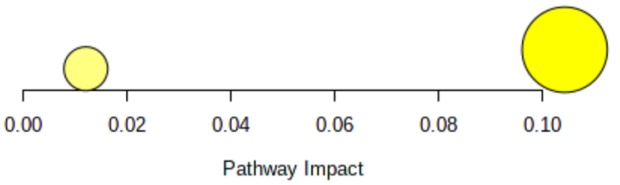

Fig. 1 Overview of the scatter plots of the metabolic pathways changed by a single dose of amphetamine $(5 \mathrm{mg} / \mathrm{kg})$ in a plasma $1 \mathrm{~h}$, b plasma $8 \mathrm{~h}$, and $\mathbf{c}$ urine $8 \mathrm{~h}$ after administration. The color of the dots is based on the negative decadic logarithm of the $p$ value. Dark color indicates a more significant pathway. The dots radius complies with the pathway impact value. Statistically significant

that may be affected by amphetamine abuse, urine is of interest for detecting metabolites (intake biomarker). The application of untargeted metabolomics to urine may allow the detection of metabolites that may be overseen by conventional pathway analysis methods because they might not be expected. Therefore, both plasma and urine were analyzed in this study to complement and confirm previous studies of the plasma metabolome after amphetamine intake and to detect additional metabolites/biomarker in urine that allow detection of amphetamine abuse. Blood draw time points of pathways $(p<0.05)$ are numbered from 1 to $6.1=$ aminoacyl-tRNAbiosynthesis; 2 = phenylalanine, tyrosine and tryptophan biosynthesis; $3=$ valine, leucine, and isoleucine biosynthesis; $4=$ ubiquinone and other terpenoid-quinone biosynthesis; $5=$ glycine, serine and threonine metabolism; $6=$ arginine biosynthesis

1,2 , and $8 \mathrm{~h}$ were chosen to examine both direct and delayed effects of amphetamines on the plasma metabolome. Blood at time point $=0$ min was not sampled to avoid additional stress to the animals prior to substance application, which could have influenced the study outcome. Furthermore, individual differences in the animals could be ruled out via the study design as changes in the metabolome between the control and amphetamine group were only assumed to be statistically significant in case they occurred in the complete group. Since the maximum plasma concentration is reached 
after $15 \mathrm{~min}$, the first two withdrawal time points were 1 and $2 \mathrm{~h}$ (Slezak et al. 2018). $8 \mathrm{~h}$ after amphetamine administration, amphetamine or metabolites of them could no longer be detected in plasma. However, effects could possibly still be detected but also some changes may also occur at a later time point and thus be undetectable (Gertsman and Barshop 2018). The two urine collection time points were chosen to be able to detect both direct and delayed effects.

It was not possible to find potential reasons for all identified altered metabolites in this study. Conclusions on a pathway can only be drawn if the pathway could be clearly identified by more than one metabolite. Metabolites that occurred as a single phenomenon of a possible pathway must, therefore, first be considered individually in their function. To be able to make specific statements about the influence of the metabolites in association with amphetamine consumption, a targeted study can be considered in which a specific analysis can be made for metabolites that occur in the proposed metabolic pathways.

\section{Plasma samples collected after controlled amphetamine administration}

The complexity of the plasma metabolome was visible comparing the PCA of the plasma datasets to the urine datasets (Fig. S9-12). Urine samples are well clustered in contrast to plasma samples regarding the multivariate statistics. This can be explained by the fact, that in contrast to plasma, most of the identified features in urine belong to amphetamine and its metabolites.

While Steuer et al. (2020) identified in human various metabolites derived from energy metabolism in general, such as acyl carnitines, fatty acids, bile acids, the current study found amino acids to be significantly changed in rat plasma. It needs to be mentioned, that the species may not be directly comparable. The difference in the results between Steuer et al. (2020) and the present study shows that comprehensive studies and different analytical strategies are necessary to study changes within the metabolome. The pathway analysis of time points 1 and $8 \mathrm{~h}$ after administration are shown in Fig. 1a and b. Except for creatine, all amino acids were downregulated in the amphetaminetreated rats compared to the control group. The pathway, which was indicated for both time points was the aminoacetyl-tRNA biosynthesis, which is an essential process in protein synthesis (Rubio Gomez and Ibba 2020). While tryptophan, histidine, methionine, threonine, and tyrosine are essential amino acids, proline and tryptophan are functional amino acids, which are important regulators of key metabolic pathways. Such pathways are necessary for maintenance growth, reproduction, and immunity in organism (Wu 2009). In addition to the amino acids, further features were identified, but these belong to MSI level 2 and were, therefore, not included in the pathway analysis. The $\mathrm{N}$-acylsphingosine ceramide (d18:1/23:0) was increased in 8-h plasma samples of amphetamine-treated rats. Ceramides are biologically used as membrane stabilizer, energy source and storage, and in inflammatory processes. The observation of amphetamine being able to increase energy metabolism also correlates with other studies conducted both in humans and in rats (Dickson 1998; Tserng and Griffin 2004). Again, species may not be comparable. Another endogenous metabolite, which is associated with the energy metabolism is tocopheronic acid (Fahy et al. 2005; Watson 2006). It is also part of the lipid metabolism and transport and was significantly decreased in comparison to the control group after $1 \mathrm{~h}$ of drug administration. Furthermore, nicotinamide was downregulated in amphetamine-treated rats. It is involved in the nicotinamide adenine dinucleotide $\left(\mathrm{NAD}^{+}\right)$signaling pathway. NAD is synthesized from both nicotinamide and degradation products of the amino acid tryptophan (Canto and Auwerx 2011). It has an important role as a cofactor in numerous metabolic processes such as glycolysis, citric acid cycle of cellular respiration, or other cellular functions (Belenky et al. 2007; Ying 2006). In plasma collected after $2 \mathrm{~h}$, only three features were identified. Two of them were identified as amphetamine and its metabolite $\mathrm{N}$-acetylamphetamine. The third feature identified was erucamide, which is an endogenous metabolite that causes reduced mobility and slightly decreased awareness in rats (Cravatt et al. 1995; McKinney and Cravatt 2005). Such oleamides could also be originating from disposable laboratory plasticware. To test whether this metabolite was a contaminant from laboratory plasticware or whether it was endogenous in origin, a study was performed according to McDonald et al. (2008) by replacing plasma with methanol. The result showed that erucamide was also found in methanol samples, but compared to plasma, the intensity and peak area was much lower. Additionally, the EIC showed a higher signal in amphetamine-treated plasma than in the control group. Therefore, it might be possible that erucamide was mainly derived from an endogenous source. All identified features except of amphetamine and its metabolite $\mathrm{N}$-acetylamphetamine were of endogenous origin and may help to understand acute and long-term effects of amphetamine abuse and are an important complement to already published results.

\section{Urine samples collected after controlled amphetamine administration}

Compared to other biofluids such as plasma, urine is characterized by being easy to collect, rich in metabolites, and able to reflect imbalances in all biochemical pathways within an organism (Khamis et al. 2017). It is, amongst others, also well suited for identifying novel exogenous drug metabolites or endogenous biomarkers indicative for drug ingestion so 
far, they are not exclusively excreted into feces. This is of particular interest for compounds, which show relatively small detection windows such as amphetamine (Carvalho et al. 2012; Kraemer and Maurer 2002; Musshoff 2000). Therefore, an untargeted metabolomics approach was used in the present study to detect endogenous and (new) exogenous metabolites in rat urine. According to previous studies in mammals (Caldwell 1976; Cho and Wright 1978; Musshoff 2000), expected amphetamine metabolites were formed mainly through (1) hydroxylation in position 4 of the aromatic ring, followed by conjugation of the phenol group with sulfate or glucuronic acid and (2) $\mathrm{N}$-deamination and oxidation into corresponding benzoic acid derivatives which were further conjugated with glycine and excreted as hippuric acids (Kraemer and Maurer 2002). However, there might be species differences to be considered. In the present study, seven amphetamine metabolites were found amongst them 4-hydroxyamphetamine and its sulfate and glucuronic acid conjugates. Features, which belong to the $N$-deamination and oxidation pathway were not indicated as statistically significant. However, six metabolites/adducts could be identified (MS ${ }^{2}$ spectra shown in Fig. S13). In detail, the $\mathrm{N}$-acetylation, which was also found in plasma samples, the $\mathrm{N}$-acetylation together with the hydroxylation of the aromatic ring, the glucuronic acid conjugate of $\mathrm{N}$-acetyl-4-hydroxyamphetamine, and the conjugate with acid succinic acid. The 6-oxohexanoic acid adduct cannot be explained from a biological point of view. It is possible that this adduct originated exogenously. However, to our knowledge this is the first report of amphetamine bound to succinic acid in rat. In addition to the amphetamine metabolites mentioned above, endogenous metabolites were also detected in urine. These included metabolites, which belong to the histamine metabolism such as $N$-acetylhistamine and histamine itself. Histamine is a powerful vasodilator, stimulant of gastric secretion, and also a centrally acting neurotransmitter. Furthermore, histamine has a considerable impact on mitigating stress-induced adverse effects in rats (Chen et al. 2020). This observation suggests that amphetamine induces additional stress to rats compared to the control group. Spermidine was decreased in amphetamine-treated rats. Polyamines such as spermidine and spermine play important roles in mammalian cells in protein and nucleic acid synthesis, protection from oxidative damage, activity of ion channels, and cell proliferation, differentiation and apoptosis (Pegg 2016). The pathway which was indicated for urine collected $8 \mathrm{~h}$ after administration was the arginine biosynthesis (Fig. 1c). Arginine, a semi-essential amino acid, is synthesized from citrulline, which has also been detected in plasma and is metabolized either to ornithine and urea or to citrulline and nitric oxide (NO). The arginine derivatives $\mathrm{N}$-acetyl-L-arginine and urea, and $N_{2}, N_{5}$-diacetylornithine, a derivate of ornithine, were also detected in urine (Cynober et al. 1995; Sasso et al. 2014). In rats, arginine acts as a key signal for the activation of ureagenesis during high-protein feeding. Additionally, arginine plays an important role in cell division, ammonia-removing from body, immune function, and hormones release. As a precursor of NO, the smallest signaling molecule in mammalian cells, arginine is thus indirectly involved in the regulation of blood pressure (Cynober et al. 1995). $\gamma$-Glutamyl- $\gamma$-aminobutyraldehyde, imidazole lactate, 5-acetylamino-6-amino-3-methyluracil, and 1,3-dimethyluracil fluctuated significantly in urine. However, the biological significance of these metabolites is currently unclear.

\section{Limitations of the study}

The present study provides only a snapshot of the metabolome in rats and a direct transfer to humans is not possible. Furthermore, individual altered features in this study could only be partly explained in terms of their general function in mammals, but not how they relate to amphetamine abuse. This is due to the fact that it is not possible to draw reliable conclusions about a specific pathway based on a single feature and thus explain processes in the organism. Thus, further studies are needed to draw reliable conclusions. However, the findings of this study may help to first understand the impact of amphetamine on the metabolome of mammals but-and this is much more of relevance- to allow a targeted design of future human studies that need then fewer subjects. Furthermore, the newly detected metabolites in rats may potentially not be formed (at least to this extent) in humans.

\section{Conclusion}

Due to the complexity of the metabolome in plasma and urine with its multitude of different metabolites, it is not possible to establish an untargeted metabolomics approach that allows a holistic view on the metabolome. For this reason, the present study is a further piece in the puzzle to elucidate, which metabolic changes occur in an organism after amphetamine intake. In this study, the major endogenous metabolites that were significantly altered belong to the compound class of amino acids. Furthermore, new amphetamine metabolites $N$-acetylamphetamine, $N$-acetyl4-hydroxyamphetamine, $\mathrm{N}$-acethyl-4-hydroxy-glucuronic amphetamine, and an amphetamine succinic acid conjugate were identified, which may be used for detection of amphetamine intake. The example of the succinate metabolite shows that untargeted metabolomics allows to identify metabolites that would otherwise not have been expected or would not have been searched for in a targeted approach. 
Supplementary Information The online version contains supplementary material available at https://doi.org/10.1007/s00204-021-03135-8.

Acknowledgements The authors would like to thank Sascha K. Manier, Carsten Schröder, Gabriele Ulrich, and Armin A. Weber for their support and/or helpful discussion.

Funding Open Access funding enabled and organized by Projekt DEAL. This research received no external funding.

Availability of data and material The R scripts and the mzXML files can be found at https://github.com/sehem/Amphetamine_Metabolomi cs.git.

\section{Declarations}

Conflict of interest The authors declare no conflict of interest.

Open Access This article is licensed under a Creative Commons Attribution 4.0 International License, which permits use, sharing, adaptation, distribution and reproduction in any medium or format, as long as you give appropriate credit to the original author(s) and the source, provide a link to the Creative Commons licence, and indicate if changes were made. The images or other third party material in this article are included in the article's Creative Commons licence, unless indicated otherwise in a credit line to the material. If material is not included in the article's Creative Commons licence and your intended use is not permitted by statutory regulation or exceeds the permitted use, you will need to obtain permission directly from the copyright holder. To view a copy of this licence, visit http://creativecommons.org/licenses/by/4.0/.

\section{References}

Adusumilli R, Mallick P (2017) Data conversion with proteowizard msconvert. Methods Mol Biol 1550:339-368. https://doi.org/10. 1007/978-1-4939-6747-6_23

Araujo AM, Carvalho M, Costa VM et al (2021) In vivo toxicometabolomics reveals multi-organ and urine metabolic changes in mice upon acute exposure to human-relevant doses of 3,4-methylenedioxypyrovalerone (MDPV). Arch Toxicol 95(2):509-527. https:// doi.org/10.1007/s00204-020-02949-2

Barnes S, Benton HP, Casazza K et al (2016) Training in metabolomics research. I. Designing the experiment, collecting and extracting samples and generating metabolomics data. J Mass Spectrom 51(7):461-475. https://doi.org/10.1002/jms.3782

Belenky P, Bogan KL, Brenner C (2007) NAD+ metabolism in health and disease. Trends Biochem Sci 32(1):12-19. https://doi.org/10. 1016/j.tibs.2006.11.006

Bonisch H, Bruss M (2006) The norepinephrine transporter in physiology and disease. Handb Exp Pharmacol 485(175):485-524. https://doi.org/10.1007/3-540-29784-7_20

Bouhifd M, Hartung T, Hogberg HT, Kleensang A, Zhao L (2013) Review: toxicometabolomics. J Appl Toxicol 33(12):1365-1383. https://doi.org/10.1002/jat.2874

Caldwell J (1976) The metabolism of amphetamines in mammals. Drug Metab Rev 5(2):219-280. https://doi.org/10.3109/03602 537609029979

Canto C, Auwerx J (2011) NAD+ as a signaling molecule modulating metabolism. Cold Spring Harb Symp Quant Biol 76:291-298. https://doi.org/10.1101/sqb.2012.76.010439
Carvalho M, Carmo H, Costa VM et al (2012) Toxicity of amphetamines: an update. Arch Toxicol 86(8):1167-1231. https://doi.org/ 10.1007/s00204-012-0815-5

Chen S, Lu D, Wang W, Chen W, Zhang S, Wei S (2020) Plasma metabolomic profiling of repeated restraint stress in rats. J Chromatogr B Analyt Technol Biomed Life Sci 1160:122294. https:// doi.org/10.1016/j.jchromb.2020.122294

Cho AK, Wright J (1978) Pathways of metabolism of amphetamine and related compounds. Life Sci 22(5):363-372. https://doi.org/ 10.1016/0024-3205(78)90282-5

Cravatt BF, Prospero-Garcia O, Siuzdak G et al (1995) Chemical characterization of a family of brain lipids that induce sleep. Science 268(5216):1506-1509. https://doi.org/10.1126/science.7770779

Cynober L, Boucher JL, Vasson M-P (1995) Arginine metabolism in mammals. J Nutr Biochem 6(8):402-413. https://doi.org/10.1016/ 0955-2863(95)00066-9

de la Torre R, Farre M, Navarro M, Pacifici R, Zuccaro P, Pichini $S$ (2004) Clinical pharmacokinetics of amfetamine and related substances: monitoring in conventional and non-conventional matrices. Clin Pharmacokinet 43(3):157-185. https://doi.org/10. 2165/00003088-200443030-00002

Dickson RC (1998) Sphingolipid functions in saccharomyces cerevisiae: comparison to mammals. Annu Rev Biochem 67:27-48. https://doi.org/10.1146/annurev.biochem.67.1.27

Dolder PC, Strajhar P, Vizeli P, Hammann F, Odermatt A, Liechti ME (2017) Pharmacokinetics and pharmacodynamics of lisdexamfetamine compared with D-amphetamine in healthy subjects. Front Pharmacol 8:617. https://doi.org/10.3389/fphar.2017.00617

Fahy E, Subramaniam S, Brown HA et al (2005) A comprehensive classification system for lipids. J Lipid Res 46(5):839-861. https:// doi.org/10.1194/jlr.E400004-JLR200

Feio-Azevedo R, Costa VM, Ferreira LM et al (2017) Toxicity of the amphetamine metabolites 4-hydroxyamphetamine and 4-hydroxynorephedrine in human dopaminergic differentiated SH-SY5Y cells. Toxicol Lett 269:65-76. https://doi.org/10.1016/j.toxlet. 2017.01.012

Gertsman I, Barshop BA (2018) Promises and pitfalls of untargeted metabolomics. J Inherit Metab Dis 41(3):355-366. https://doi.org/ 10.1007/s10545-017-0130-7

Guijas C, Montenegro-Burke JR, Domingo-Almenara X et al (2018) METLIN: a technology platform for identifying knowns and unknowns. Anal Chem 90(5):3156-3164. https://doi.org/10.1021/ acs.analchem. $7 \mathrm{~b} 04424$

Heal DJ, Smith SL, Gosden J, Nutt DJ (2013) Amphetamine, past and present-a pharmacological and clinical perspective. J Psychopharmacol 27(6):479-496. https://doi.org/10.1177/0269881113 482532

Hemmer S, Manier SK, Fischmann S, Westphal F, Wagmann L, Meyer MR (2020) Comparison of three untargeted data processing workflows for evaluating LC-HRMS metabolomics data. Metabolites. https://doi.org/10.3390/metabo10090378

Khamis MM, Adamko DJ, El-Aneed A (2017) Mass spectrometric based approaches in urine metabolomics and biomarker discovery. Mass Spectrom Rev 36(2):115-134. https://doi.org/10.1002/ mas. 21455

Kind T, Liu KH, Lee DY, DeFelice B, Meissen JK, Fiehn O (2013) LipidBlast in silico tandem mass spectrometry database for lipid identification. Nat Methods 10(8):755-758. https://doi.org/10. 1038/nmeth.2551

Kraemer T, Maurer H (2002) Toxicokinetics of amphetamines: metabolism and toxicokinetic data of designer drugs, amphetamine, methamphetamine, and their $\mathrm{N}$-alkyl derivatives. Ther Drug Monit 24(2):277-289

Kuhl C, Tautenhahn R, Bottcher C, Larson TR, Neumann S (2012) CAMERA: an integrated strategy for compound spectra extraction and annotation of liquid chromatography/mass spectrometry 
data sets. Anal Chem 84(1):283-289. https://doi.org/10.1021/ ac202450g

Linstrom PJ, Mallard WG (2001) The NIST chemistry webbook: a chemical data resource on the internet $\uparrow$. J Chem Eng Data 46(5):1059-1063. https://doi.org/10.1021/je000236i

Liu X, Locasale JW (2017) Metabolomics: a primer. Trends Biochem Sci 42(4):274-284. https://doi.org/10.1016/j.tibs.2017.01.004

Manier SK, Meyer MR (2020) Impact of the used solvent on the reconstitution efficiency of evaporated biosamples for untargeted metabolomics studies. Metabolomics 16(3):34. https://doi.org/10. 1007/s11306-019-1631-1

Manier SK, Keller A, Meyer MR (2019a) Automated optimization of XCMS parameters for improved peak picking of liquid chromatography-mass spectrometry data using the coefficient of variation and parameter sweeping for untargeted metabolomics. Drug Test Anal 11(6):752-761. https://doi.org/10.1002/dta.2552

Manier SK, Keller A, Schaper J, Meyer MR (2019b) Untargeted metabolomics by high resolution mass spectrometry coupled to normal and reversed phase liquid chromatography as a tool to study the in vitro biotransformation of new psychoactive substances. Sci Rep 9(1):2741. https://doi.org/10.1038/s41598-019-39235-w

Manier SK, Schwermer F, Wagmann L, Eckstein N, Meyer MR (2020a) Liquid chromatography-high-resolution mass spectrometry-based in vitro toxicometabolomics of the synthetic cathinones 4-MPD and 4-MEAP in pooled human liver microsomes. Metabolites. https://doi.org/10.3390/metabo11010003

Manier SK, Wagmann L, Flockerzi V, Meyer MR (2020b) Toxicometabolomics of the new psychoactive substances alpha-PBP and alpha-PEP studied in HepaRG cell incubates by means of untargeted metabolomics revealed unexpected amino acid adducts. Arch Toxicol 94(6):2047-2059. https://doi.org/10.1007/ s00204-020-02742-1

Maurer HH, Pfleger K, Weber AA (2016) Mass spectral data of drugs, poisons, pesticides, pollutants and their metabolites. Wiley-VCH, Weinheim

Maurer HH, Meyer MR, Helfer AG, Weber AA (2018) Maurer/Meyer/ Helfer/Weber MMHW LC-HR-MS/MS library of drugs, poisons, and their metabolites. Wiley-VCH, Weinheim

McDonald GR, Hudson AL, Dunn SM et al (2008) Bioactive contaminants leach from disposable laboratory plasticware. Science 322(5903):917. https://doi.org/10.1126/science.1162395

McKinney MK, Cravatt BF (2005) Structure and function of fatty acid amide hydrolase. Annu Rev Biochem 74:411-432. https://doi.org/ 10.1146/annurev.biochem.74.082803.133450

Musshoff F (2000) Illegal or legitimate use? Precursor compounds to amphetamine and methamphetamine. Drug Metab Rev 32(1):1544. https://doi.org/10.1081/dmr-100100562

Nair AB, Jacob S (2016) A simple practice guide for dose conversion between animals and human. J Basic Clin Pharm 7(2):27-31. https://doi.org/10.4103/0976-0105.177703

Nicholson JK, Lindon JC (2008) Systems biology: metabonomics. Nature 455(7216):1054-1056. https://doi.org/10.1038/4551054a

Pegg AE (2016) Functions of polyamines in mammals. J Biol Chem 291(29):14904-14912. https://doi.org/10.1074/jbc.R116.731661

Psychoaktivesubstanzen.de/amphetamin/Amphetamin/Speed/AmpheBekannter, verbotener Upper (Substanzinfo). In. http://psychoakti vesubstanzen.de/amphetamin/. Accessed 26 May 2020, 9:30

Ramirez T, Daneshian M, Kamp H et al (2013) Metabolomics in toxicology and preclinical research. Altex 30(2):209-225. https://doi. org/10.14573/altex.2013.2.209

Rubio Gomez MA, Ibba M (2020) Aminoacyl-tRNA synthetases. RNA 26(8):910-936. https://doi.org/10.1261/rna.071720.119

Sasso S, Dalmedico L, Delwing-Dal Magro D, Wyse AT, Delwingde Lima D (2014) Effect of $\mathrm{N}$-acetylarginine, a metabolite accumulated in hyperargininemia, on parameters of oxidative stress in rats: protective role of vitamins and L-NAME. Cell Biochem Funct 32(6):511-519. https://doi.org/10.1002/cbf.3045
Slezak JM, Mueller M, Ricaurte GA, Katz JL (2018) Pharmacokinetic and pharmacodynamic analysis of d-amphetamine in an attention task in rodents. Behav Pharmacol 29(6):551-556. https://doi.org/ 10.1097/FBP.0000000000000409

Smith CA, Want EJ, O’Maille G, Abagyan R, Siuzdak G (2006) XCMS: processing mass spectrometry data for metabolite profiling using nonlinear peak alignment, matching, and identification. Anal Chem 78(3):779-787. https://doi.org/10.1021/ac051437y

Steinkellner T, Freissmuth M, Sitte HH, Montgomery T (2011) The ugly side of amphetamines: short- and long-term toxicity of 3,4-methylenedioxymethamphetamine (MDMA, 'Ecstasy'), methamphetamine and D-amphetamine. Biol Chem 392(1-2):103-115. https://doi.org/10.1515/BC.2011.016

Steuer AE, Brockbals L, Kraemer T (2019) Metabolomic strategies in biomarker research-new approach for indirect identification of drug consumption and sample manipulation in clinical and forensic toxicology? Front Chem 7:319. https://doi.org/10.3389/fchem. 2019.00319

Steuer AE, Kaelin D, Boxler MI et al (2020) Comparative untargeted metabolomics analysis of the psychostimulants 3,4-methylenedioxy-methamphetamine (MDMA), amphetamine, and the novel psychoactive substance mephedrone after controlled drug administration to humans. Metabolites. https://doi.org/10.3390/metab o10080306

Sumner LW, Amberg A, Barrett D et al (2007) Proposed minimum reporting standards for chemical analysis chemical analysis working group (CAWG) metabolomics standards initiative (MSI). Metabolomics 3(3):211-221. https://doi.org/10.1007/ s11306-007-0082-2

Team RC R: A language and environment for statistical computing. 3.4.1 edn. R Foundation for Statistical Computing

Tserng KY, Griffin RL (2004) Ceramide metabolite, not intact ceramide molecule, may be responsible for cellular toxicity. Biochem J 380(Pt 3):715-722. https://doi.org/10.1042/BJ20031733

UNODC (2020) World drug report 2020-booklet 1. United Nations Publication, New York

Wang L, Wu N, Zhao TY, Li J (2016) The potential biomarkers of drug addiction: proteomic and metabolomics challenges. Biomarkers 21(8):678-685. https://doi.org/10.1080/1354750X.2016.1201530

Watson AD (2006) Thematic review series: systems biology approaches to metabolic and cardiovascular disorders. Lipidomics: a global approach to lipid analysis in biological systems. J Lipid Res 47(10):2101-2111. https://doi.org/10.1194/jlr.R6000 22-JLR200

Wehrens R, Hageman JA, van Eeuwijk F et al (2016) Improved batch correction in untargeted MS-based metabolomics. Metabolomics 12:88. https://doi.org/10.1007/s11306-016-1015-8

Wishart DS, Tzur D, Knox C et al (2007) HMDB: the human metabolome database. Nucleic Acids Res 35:D521-D526. https://doi. org/10.1093/nar/gk1923

Wu G (2009) Amino acids: metabolism, functions, and nutrition. Amino Acids 37(1):1-17. https://doi.org/10.1007/ s00726-009-0269-0

Ying W (2006) NAD+ and NADH in cellular functions and cell death. Front Biosci 11:3129-3148. https://doi.org/10.2741/2038

Zaitsu K, Hayashi Y, Kusano M, Tsuchihashi H, Ishii A (2016) Application of metabolomics to toxicology of drugs of abuse: a mini review of metabolomics approach to acute and chronic toxicity studies. Drug Metab Pharmacokinet 31(1):21-26. https://doi.org/ 10.1016/j.dmpk.2015.10.002

Publisher's Note Springer Nature remains neutral with regard to jurisdictional claims in published maps and institutional affiliations. 